\title{
Homogenization of an Ensemble of Swiss Rolls by means of the MLFMA
}

B. Michiels*

I. Bogaert*

\begin{abstract}
-
This paper shows the homogenization of a magnetic metamaterial, consisting of so-called Swiss rolls, using MLFMA simulations. First, the resonance frequency of a single Swiss roll is determined. Next, a bianisotropic model is used to determine the macroscopic material parameters of an ensemble of Swiss rolls by S-parameter retrieval. Finally, the macroscopic material parameters (the permeability, permittivity and magnetoelectric coupling coefficients) are found as a function of frequency.
\end{abstract}

\section{INTRODUCTION}

In general, metamaterials consist of microscopic structures and these microscopic structures can be designed to construct materials with remarkable properties, e.g. a negative refractive index. However, the retrieval of meaningful material parameters from these structures is often a hard problem to tackle.

In this paper, the metamaterial structure of interest is the so-called Swiss roll, which is a rolledup, perfectly electrically conducting (PEC), infinitely long sheet [1]. When an incident transverseelectric (TE) polarized wave impinges on a Swiss roll, a current along the surface of the Swiss roll is induced and the magnetic field in the center shows resonant behavior as a function of frequency.

This paper presents simulations of Swiss rolls and the determination of the macroscopic material parameters of an ensemble of Swiss rolls. To perform the simulations, boundary integral equations are used and discretized by the Method of Moments (MoM). To reduce the simulation time, a low-frequency stable MultiLevel Fast Multipole Algorithm (MLFMA) [2] is applied.

\section{SWISS ROLLS}

The geometry of the Swiss roll has many degrees of freedom, which will all have an influence on the behavior of the metamaterial, e.g. the resonance frequency. The Swiss rolls, considered in this paper, have, by way of example, 6 windings $(W)$, a maximal radius of $1 \mathrm{~m}\left(R_{\max }\right)$, a distance between the plates of $5 \mathrm{~cm}\left(\Delta_{1} R_{\max }\right)$ and a plate thickness

\footnotetext{
*Department of Information Technology (INTEC), Ghent University, Ghent, B-9000, Belgium http://emweb.intec.ugent.be
}
J. Fostier*
D. De Zutter*

of $5 \mathrm{~cm}\left(\Delta_{2} R_{\max }\right)$. The parametric equation of a Swiss roll is:

$$
\begin{gathered}
{[x, y]=R(\phi)[\cos \phi, \sin \phi]} \\
R^{\text {outer }}(\phi)=R_{\max }\left(1-\left(\Delta_{1}+\Delta_{2}\right) \frac{\phi}{2 \pi}\right), \\
\phi=[0 \ldots 2 \pi W] \\
R^{\text {inner }}(\phi)=R_{\max }\left(1-\Delta_{2}-\left(\Delta_{1}+\Delta_{2}\right) \frac{\phi}{2 \pi}\right), \\
\phi=[2 \pi W \ldots 0]
\end{gathered}
$$

To determine the resonance frequency, simulations with one Swiss roll are performed and as an excitation a TE-polarized plane wave, propagating along the positive $x$-axis $\left(H_{z}^{\text {in }}=H_{0} e^{-j k_{0} x}\right)$ is used. The resonance frequency can be found from a frequency sweep, with frequency steps of $\Delta f$, shown in Fig. 1. A discretization of $\Delta \phi=0.1$ (756 segments) is used, which yields accurate results for the considered geometry [3].

Fig. 1 shows the total magnetic field in the center of the Swiss roll, normalized to the amplitude of the incident plane wave. The resonance frequency, i.e. the frequency for which the magnetic field reaches

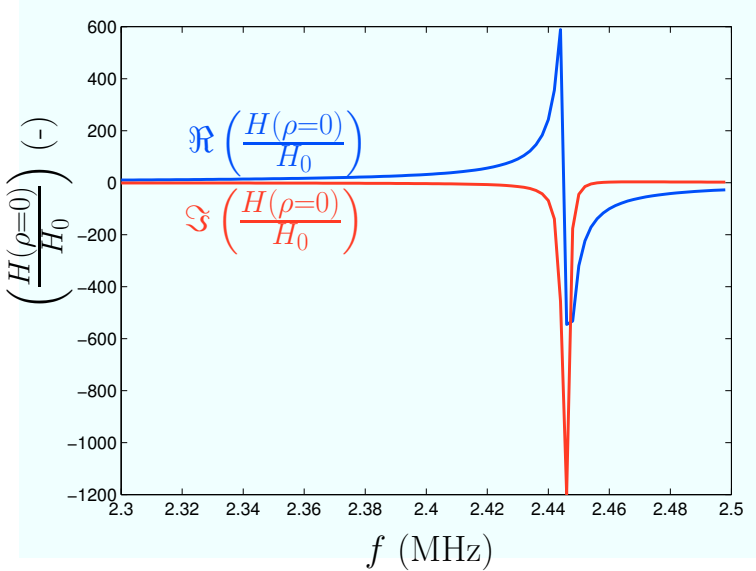

Figure 1: Frequency sweep for $\Delta f=2 \mathrm{kHz}$. The magnetic field in the center of the Swiss roll is normalized to the amplitude of the incident magnetic field. 
its maximum, for the considered Swiss roll geometry is equal to $2.446 \mathrm{MHz}$.

\section{PARALLEL-PLATE SET-UP}

WAVEGUIDE

To determine the equivalent macroscopic material parameters, the S-parameters of a Swiss roll ensemble can be fitted to the S-parameters caused by scattering at a homogeneous medium. As a simulation configuration, we consider a single row of Swiss rolls put into a parallel-plate waveguide, which strongly reduces the required computational capacity with respect to a configuration that uses large grids. Using image theory, this waveguide setup can be converted into the equivalent layered media problem (bottom of Fig. 2).

The centers of the Swiss rolls are located at $x=\left(n-\frac{1}{2}\right) d$ (with $\left.n=1 \ldots N\right)$, with $N$ the total number of Swiss rolls in the waveguide and $d$ the lattice constant of the row of Swiss rolls (chosen equal to $3 \mathrm{~m}$ ). Due to symmetry reasons, the boundaries of the homogenized medium should be located at $x=0$ and $x=N d[3]$.

\section{BIANISOTROPIC MODEL}

To model the homogenized ensemble of Swiss rolls, a bianisotropic model will be used. We start from

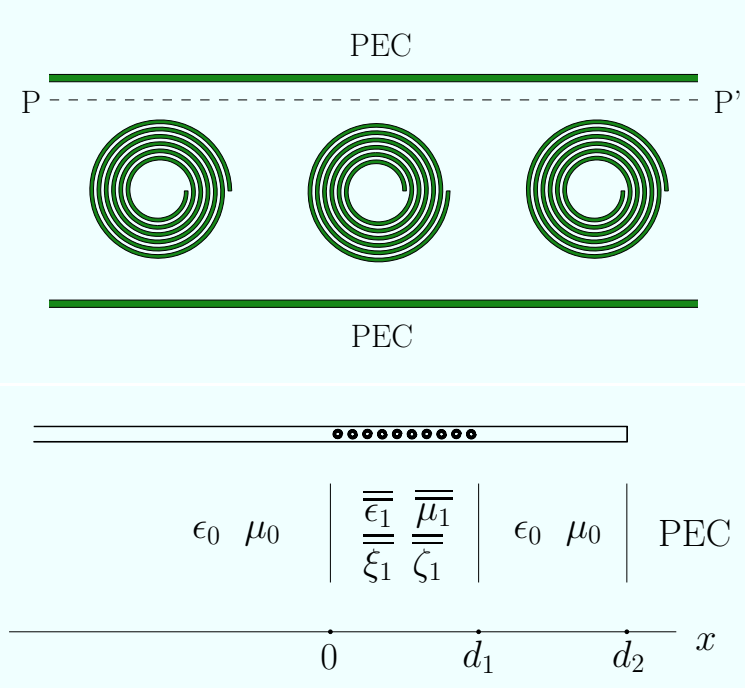

Figure 2: Swiss rolls stacked into a waveguide. Using image theory, this problem can be converted into a layered media problem. the following general constitutive equations:

$$
\begin{aligned}
& \bar{D}=\overline{\bar{\epsilon}} \cdot \bar{E}+\overline{\bar{\xi}} \cdot \bar{H} \\
& \bar{B}=\overline{\bar{\zeta}} \cdot \bar{E}+\overline{\bar{\mu}} \cdot \bar{H}
\end{aligned}
$$

with $\overline{\bar{\epsilon}}$, the permittivity tensor, $\overline{\bar{\mu}}$, the permeability tensor and $\overline{\bar{\xi}}$ and $\overline{\bar{\zeta}}$, the magnetoelectric coupling pseudotensors.

Due to symmetry reasons and considering the incident TE-polarization, only the following elements of the tensors and pseudotensors of formula (2) come into play: $\epsilon_{y y}, \mu_{z z}, \xi_{y z}$ and $\zeta_{z y}$ [3]. In this paper the values of these physical quantities are normalized to $\epsilon_{0}, \mu_{0}, \frac{1}{c}$ and $\frac{1}{c}$ respectively.

In the general case, the wavenumber and impedance can be different for both directions: $k_{x}^{ \pm}$ and $Z^{ \pm}$for the propagation along the positive and negative $x$-axis respectively. The relationship between the wavenumbers, impedances and the material parameters is:

$$
\begin{aligned}
\epsilon_{y y} & =\frac{Z_{0}\left(k_{x}^{+}+k_{x}^{-}\right)}{k_{0}\left(Z^{+}+Z^{-}\right)} \\
\mu_{z z} & =\frac{Z^{+} Z^{-}\left(k_{x}^{+}+k_{x}^{-}\right)}{k_{0} Z_{0}\left(Z^{+}+Z^{-}\right)} \\
\xi_{y z} & =\frac{k_{x}^{+} Z^{-}-k_{x}^{-} Z^{+}}{k_{0}\left(Z^{+}+Z^{-}\right)} \\
\zeta_{z y} & =\frac{k_{x}^{+} Z^{+}-k_{x}^{-} Z^{-}}{k_{0}\left(Z^{+}+Z^{-}\right)}
\end{aligned}
$$

Now the bianisotropic model can be applied to the homogenized medium of the waveguide set-up in Fig. 2. When the excitation is a TE-polarized plane wave, we can write the electromagnetic fields as:

$$
\begin{aligned}
x<0: H_{z} & =A e^{-j k_{0} x}+B e^{j k_{0} x} \\
E_{y} & =Z_{0}\left(A e^{-j k_{0} x}-B e^{j k_{0} x}\right) \\
0<x<d_{1}: H_{z} & =C e^{-j k_{1}^{+} x}+D e^{j k_{1}^{-} x} \\
E_{y} & =Z_{1}^{+} C e^{-j k_{1}^{+} x}-Z_{1}^{-} D e^{j k_{1}^{-} x} \\
d_{1}<x<d_{2}: H_{z} & =F e^{-j k_{0} x}+G e^{j k_{0} x} \\
E_{y} & =Z_{0}\left(F e^{-j k_{0} x}-G e^{j k_{0} x}\right)
\end{aligned}
$$

From the simulations performed on the waveguide set-up, one can obtain the S-parameters $A, B$, $F$ and $G$. However, applying the boundary conditions at $x=0$ and $x=d_{1}$ would lead to only 4 equations for 6 unknowns, i.e. $k_{1}^{ \pm}, Z_{1}^{ \pm}, C$ and $D$. Therefore we also have to consider a second simulation, where the Swiss rolls are rotated over an angle $\pi \cdot \overline{\bar{\xi}}$ and $\overline{\bar{\zeta}}$ are pseudotensors, so this operation results in 
the transformations $\xi_{y z} \rightarrow-\xi_{y z}$ and $\zeta_{z y} \rightarrow-\zeta_{z y}$. From formula (3) it follows that changing the signs of $\xi_{y z}$ and $\zeta_{z y}$ corresponds with the transformation $\left(k_{1}^{+}, k_{1}^{-}, Z_{1}^{+}, Z_{1}^{-}\right) \rightarrow\left(k_{1}^{-}, k_{1}^{+}, Z_{1}^{-}, Z_{1}^{+}\right)$.

Finally, applying the boundary conditions of both simulations at $x=0$ and $x=d_{1}$ leads to 8 equations for 8 unknowns, i.e. $k_{1}^{ \pm}, Z_{1}^{ \pm}, C^{1 / 2}$ and $D^{1 / 2}$, where the superscript " 1 " and " 2 " stands for the first and second simulation respectively.

\section{MATERIAL PARAMETERS}

Now the theory of the previous section can be applied to our Swiss roll example. We restrict ourselves to a frequency range around the resonance frequency, from $f=2.356 \mathrm{MHz}$ to $f=2.556$ $\mathrm{MHz}$ in steps of $1 \mathrm{kHz}$. Fig. 3 displays the obtained macroscopic permeability and permittivity for $N=4$ (small circles) and $N=8$ (full line).

As one can see from Fig. $3, \mu_{z z}$ and $\epsilon_{y y}$ have no imaginary part, while $\xi_{y z}$ and $\zeta_{z y}$ are purely imaginary numbers. The obtained permeability and permittivity satisfy passivity $(\Im(\mu) \leqslant 0$ and $\Im(\epsilon) \leqslant 0)$ and causality $(\partial \Re(\mu) / \partial \omega \geqslant 0$ and $\partial \Re(\epsilon) / \partial \omega \geqslant 0)$, as shown in Fig. 3(a). From Fig. 3(b) one sees that $\zeta_{z y}=-\xi_{y z}$, which implies that $k_{x}^{+}=k_{x}^{-}$(formula (3)) and confirms the reciprocity of the Swiss roll metamaterial.

It is important to remark that the curves show physically incorrect peaks, for $N=4$ Swiss rolls at $f=2.434 \mathrm{MHz}$ and for $N=8$ Swiss rolls at $f=2.384 \mathrm{MHz}$ and $f=2.434 \mathrm{MHz}$. For these frequencies the wavelength inside the homogenized Swiss roll medium equals $\frac{2 d_{1}}{n}$, so no information about the impedances can be obtained from the boundary conditions at $x=0$ and $x=d_{1}$.

\section{CONCLUSION}

In this paper Swiss rolls are investigated using fullwave simulations. First, we determined the resonance frequency of a single Swiss roll. Next, the macroscopic material parameters of an ensemble of Swiss rolls are obtained by S-parameter retrieval, using a bianisotropic model. Therefore a single row of Swiss rolls is put into a parallel-plate waveguide, which strongly reduces the required computational capacity with respect to large grids. The obtained macroscopic permeability, permittivity and magnetoelectric coupling coefficients are passive, causal and reciproque.

\section{Acknowledgment}

The computational resources (Stevin Supercomputer Infrastructure) and services used in this work

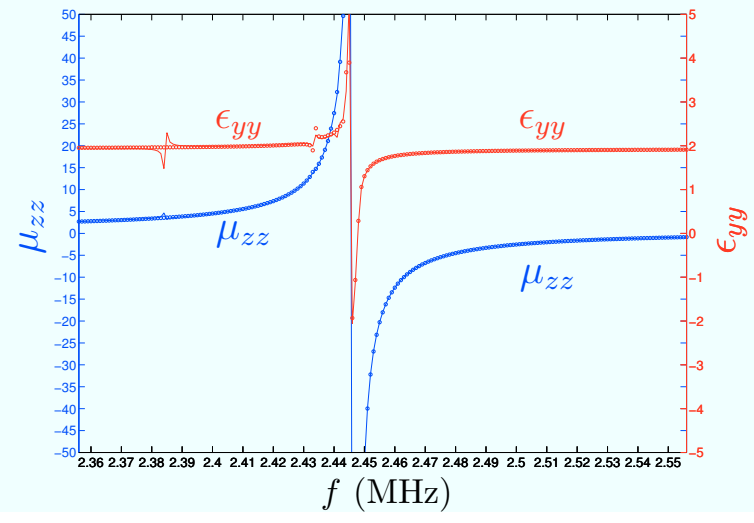

(a) Relative permeability (blue) and permittivity (red).

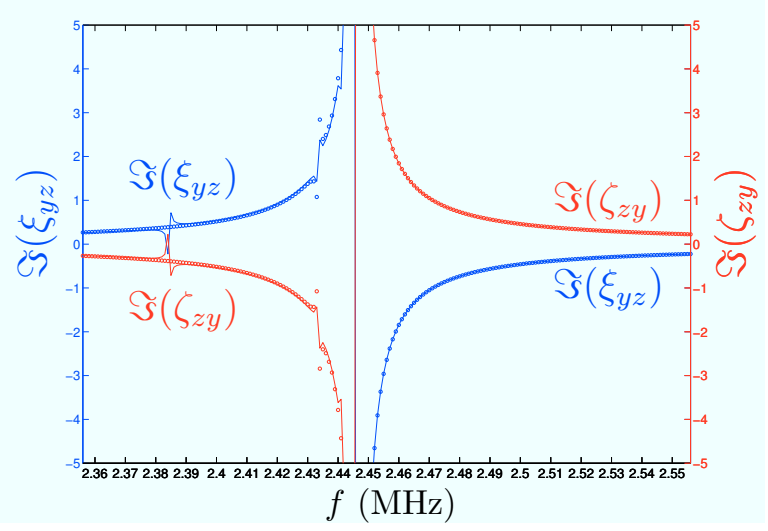

(b) Magnetoelectric coupling coefficients: $\xi_{y z}$ (blue) and $\zeta_{z y}$ (red).

Figure 3: Material parameters as a function of frequency for $N=4$ (circles) and $N=8$ (full line) Swiss rolls.

were provided by Ghent University.

\section{References}

[1] J. Pendry, A. Holden, D. Robbins, W. Stewart, "Magnetism from Conductors and Enhanced Nonlinear Phenomena", IEEE Transactions on Microwave Theory ad Techniques, vol. 47, no. 11, pp. 2075-2084, November 1999.

[2] B. Michiels, I. Bogaert, J. Fostier, J. Peeters and D. De Zutter, "Simulation of a Luneburg Lens using a Broadband MLFMA", Radio Science, vol. 46, March 2011.

[3] B. Michiels, I. Bogaert, J. Fostier and D. De Zutter, "Swiss Roll Ensemble Homogenization by Full-Wave Simulations", Microwave and Optical Technology Letters, accepted for publication, 2011. 